\title{
Negligência unilateral pós-acidente vascular encefálico: atuaçáo da Terapia Ocupacional ${ }^{1}$
}

\author{
Tamara Pereira de Oliveira ${ }^{\mathrm{a}, \mathrm{b}}$, Rita de Cássia Tibério Araújo ${ }^{\mathrm{c}}$, Edvaldo Soares $^{\mathrm{d}, \mathrm{e}}$

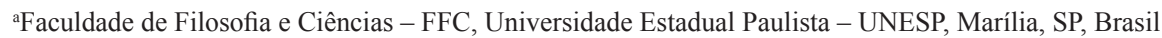

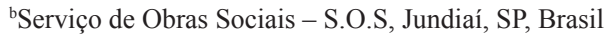 \\ 'Departamento de Fisioterapia e Terapia Ocupacional, Faculdade de Filosofia e Ciências - FFC, \\ Universidade Estadual Paulista - UNESP, Marília, SP, Brasil \\ dUniversidade de São Paulo - USP, São Paulo, SP, Brasil \\ eDepartamento de Psicologia da Educação, Faculdade de Filosofia e Ciências - FFC, \\ Universidade Estadual Paulista - UNESP, Marília, SP, Brasil
}

\begin{abstract}
Resumo: A negligência unilateral é uma das consequências do acidente vascular encefálico e geralmente resulta de lesão no lobo parietal direito, afetando as funções perceptivas visuais, espaciais e de atenção. No âmbito da funcionalidade, o paciente acometido tem dificuldade para perceber os estímulos do ambiente dispostos do lado contralesional. Dentre as abordagens terapêuticas, as intervenções na área de Terapia Ocupacional constituem parte do tratamento desse paciente para a melhora do desempenho nas atividades de vida. Neste estudo, deu-se ênfase à descrição do processo de intervenção de Terapia Ocupacional, objetivando-se identificar o progresso do paciente com base no contínuo do seu quadro evolutivo. A pesquisa foi realizada com sujeito único em atendimento terapêutico ocupacional. Os dados foram obtidos por meio de levantamento dos registros de prontuário do paciente e de entrevistas realizadas com o paciente, sua terapeuta e sua cuidadora, a fim de descrever o atendimento e seus resultados. $\mathrm{Na}$ análise dos registros de prontuário e dos relatos da terapeuta verificou-se que o paciente respondeu satisfatoriamente ao tratamento, apresentando diminuição da negligência unilateral à esquerda ao final do período estudado. O impacto favorável do tratamento segundo relatos de entrevista indica a satisfação do paciente em participar da Terapia Ocupacional e a alegria com a vida. Diante dos resultados alcançados considera-se relevante a exploração de práticas sistematizadas de intervenção na área de Terapia Ocupacional com o intuito de investigar a eficácia da intervenção perante quadros clínicos de negligência unilateral.
\end{abstract}

Palavras-chave: Acidente Vascular Encefálico, Terapia Ocupacional, Reabilitação.

\section{Unilateral neglect syndrome after stroke: the role of Occupational Therapy}

\begin{abstract}
Unilateral Neglect Syndrome is one of the consequences of cerebral vascular accident (CVA) generally following right parietal lobe lesion, leading to the impairment of perceptive visual, spatial and attention functions. The patient affected does not realize the environmental stimuli on the contralesional hemibody. Occupational therapy plays an important role in caring for this patient, seeking the recovery of perception, attention and social engagement. This study aimed to describe and evaluate the results of occupational therapy intervention and treatment in a single Unilateral Neglect Syndrome post CVA patient. Data were obtained from a survey of the patient's medical records and interviews of his therapist and caretaker. The analysis of the patient's medical records and his therapist's report showed that the patient responded satisfactorily to treatment, presenting a decrease of the left unilateral neglect at the end of the study period. The favorable outcome of the patient outlined the relevance of evaluating the effects of Occupational Therapy interventions for clinical unilateral neglect syndrome.
\end{abstract}

Keywords: Stroke, Occupational Therapy, Rehabilitation.

Autor para correspondência: Edvaldo Soares, Faculdade de Filosofia e Ciências, Universidade Estadual Paulista, Av. Hygino Muzzi Filho, 737 , Campus Universitário, CEP 17525-000, Marília, SP, Brasil, e-mail: edsoares@ marilia.unesp.br

Recebido em 12/6/2012; Revisão em 14/11/2012; Aceito em 7/12/12. 


\section{Introdução}

Embora a expectativa de vida da população mundial cresça, estudos apontam para a alta incidência de patologias vasculares, especialmente de doenças cerebrovasculares (SAPOSNIK; DEL BRUTO, 2003; LESSA, 1999). O acidente vascular encefálico (AVE) é uma doença cerebrovascular considerada a segunda principal causa de morte e a principal causa de incapacidades físicas e cognitivas em países desenvolvidos e em desenvolvimento (DOYLE, 2002; SARTI et al., 2000; LESSA, 1999; CABRAL et al., 1997).

Há uma menor ocorrência de AVE em indivíduos abaixo de 50 anos (STOKES, 2000). Entretanto, observa que a incidência aumenta progressivamente nas faixas etárias subsequentes, dobrando a cada década após os 55 anos de idade (GILES; ROTHWELL, 2008; RODGERS, 2004).

A ocorrência de um AVE gera transformaçóes na vida cotidiana e na participação social, tanto da pessoa como da família (MENDONÇA; GARANHANI; MARTINS, 2008; LAVINSKY; VIEIRA, 2004), requerendo a ampliação de programas de intervençáo na saúde pública (SILVA, 2004; SAPOSNIK; DEL BRUTO, 2003; SCHAU et al., 2003; PETRILLI et al., 2002; BONITA; SOLOMON; BROAD, 1997). O desfecho neurológico da interrupção do fluxo sanguíneo para o cérebro depende da etiologia, da localizaçáo e do tamanho da isquemia ou da hemorragia (LUNDY-EKMAN, 2004). As sequelas após um AVE são diversas, dentre elas, uma das mais frequentes é a heminegligência (HN) ou Síndrome de Negligência Unilateral (SNU) (NAPOLITANO; TRIACA, 2007; MOURA; SILVA, 2005; PLUMMER; MORRIS; DUNAI, 2003).

A negligência unilateral (NU) pode ser definida como dificuldade em se orientar em direção a algo localizado espacialmente ou de resposta a estímulos gerados do lado contralateral à lesão cortical, caracterizando-se pela inabilidade de o sujeito registrar, integrar ou responder a eventos provenientes do hemicorpo ou hemiespaço contralateral à lesão cerebral (NAPOLITANO; TRIACA, 2007; CAMPOS; SANTOS; XAVIER, 1997). O indivíduo com NU apresenta prejuízo na percepçáo de uma das metades do espaço egocêntrico contralateral ao hemisfério cerebral comprometido (BEAR; CONNORS; PARADISO, 2006; SANTOMÉ; MARTÍN-LOECHES; FRANK, 1998; HEILMAN; WATSON; VALENSTEIN, 1993; ROBERTSON; MARSHALL, 1993).
São variados os tipos de heminegligência, conforme a localização da lesão cerebral. De forma geral, a NU pode ser classificada em negligência sensorial (ausência de atenção), negligência motora (desordem de ação e intenção de movimento) e em desordens de representação mental (MESULAM, 1999; HEILMAN; VALENSTEIN; WATSON, 1994; HEILMAN; WATSON; VALENSTEIN, 1993).

Outra possibilidade de classificação, incluindo funçôes visuais e táteis, apresenta a seguinte organização da manifestação: 1) Hemi-inatenção, quando não há respostas a estímulos do lado contralateral a lesão; 2) Negligência visual, quando o paciente não reconhece estímulos visuais no hemiespaço negligente; 3) Extinção tátil e visual, quando o indivíduo é estimulado bilateralmente e só reconhece o hemicorpo não afetado; e 4) Hipocinesia ou extinçâo motora, quando se observa uma sensível diminuição da movimentação do hemicorpo acometido (CARR; SHEPHERD, 2008; SILVA; MARTINS, 2000).

Uma terceira possibilidade consiste na classificação da negligência em: negligência espacial e negligência pessoal. Na negligência espacial, o paciente negligencia o hemiespaço contralesional. Segundo Lundy-Ekman (2008), esse tipo de negligência dificulta à pessoa localizar-se dentro de uma única sala. Na negligência pessoal, o paciente náo reconhece o lado corporal contralesional. O paciente que náo explora um lado do espaço pode apresentar: deficiência na exploração visual; perda do esquema corporal; hemi-inatenção dos estímulos táteis, auditivos e visuais e incapacidade para iniciar movimentos (GRIEVE, 2006; LOVO, 2006).

Uma característica proeminente dessa síndrome é a extinção, a falha em perceber ou responder ao estímulo contralesional quando apresentado simultaneamente com um estímulo ipsilesional. O maior comprometimento cognitivo se refere basicamente aos mecanismos de atenção (SWAN, 2001; MESULAM, 1994, 1985), os quais ocasionam comprometimento de outras funçóes cognitivas como, por exemplo, a memória, gerando sintomas com diferentes manifestações (FONSECA et al., 2006; HELENE; XAVIER, 2003; PIERCE; BUXBAUM, 2002; BAILEY; RIDDOCH, 1999). Em relação à memória, é importante salientar que a NU não está relacionada diretamente com déficits de memória, mas seria fruto de um prejuízo de atenção para a porção do espaço contralateral ao hemisfério lesado, decorrente de sua hipoatividade (HEILMAN; WATSON; VALENSTEIN, 1993). Em outras palavras, o nível de atividade do hemisfério íntegro 
seria proporcionalmente maior do que o do lesado, deslocando assim o foco de atenção (LUNDYEKMAN, 2008; CAMPOS; SANTOS; XAVIER, 1997).

A incapacidade de orientação da atenção relaciona-se também às dificuldades quanto à: alimentação, vestuário, higiene, leitura, havendo uma distorção na forma como a pessoa percebe o ambiente extrapessoal e a si mesma (MARK, 2003).

Embora a NU possa ocorrer em doenças que envolvem lesão cerebral estrutural, ela tem sido mais frequentemente avaliada e estudada após AVE e tem sido associada com prejuízos na recuperação funcional após lesão cerebral com maior frequência do que outras alterações cognitivas (MARK, 2003).

No âmbito dos prejuízos funcionais decorrentes das lesôes do lobo parietal, os distúrbios da formação da imagem corporal e da percepção da relação do corpo com o espaço externo podem influenciar dramaticamente a qualidade de vida da pessoa, interferindo diretamente na sua participação social (GAZZANIGA; IVRY; MANGUN, 2006; GAZZANIGA; HEATHERTON, 2005; KANDEL; SCHWARTZ; JESSEL, 2000).

Apesar de ser descrita como um fenômeno homogêneo, a NU inclui uma série de distúrbios, os quais podem ter diferentes bases neurofisiológicas, dificultando o desenvolvimento de padróes para diagnosticar, reportar e avaliar a NU (CUBELLI et al., 1991). Diante dessa variabilidade e da diversidade de histórias de vida, justifica-se a realização de estudos com delineamento metodológico direcionado para a investigação de sintomas e de resultados de tratamento.

No tratamento da NU não basta identificar a presença ou ausência da NU em diferentes tarefas, também é preciso compreender quais características da tarefa alteram a magnitude da negligência. E esse é um direcionamento que norteia as intervençóes realizadas na área de Terapia Ocupacional, abrangendo o diagnóstico da funcionalidade sob aspectos das habilidades do desempenho e das demandas das atividades (AMERICAN..., 2010).

Com base nas áreas afetadas, foram desenvolvidos vários instrumentos de avaliação para NU na Neurociência. Entre eles: Tarefas de Bissecção de Linhas, Testes de Extinção e Confrontação, Atenção por Cancelamento, Desenho, Catherine Bergego Scale (CBS), Teste do Relógio e o Teste de Desatenção Comportamental (BIT) (CARR; SHEPHERD, 2008; CARDOSO et al., 2005; GUIMARÃES; GUIMARÃES, 2004; PLUMMER;
MORRIS; DUNAI, 2003; HARTMAN-MAEIR; KATZ, 1995).

Os testes de Atenção por Cancelamento exigem a capacidade de localizar e cancelar ou marcar os estímulos do alvo a partir de uma série de estímulos, que podem ser apresentados ao acaso ou de forma estruturada, em formação linear, incluindo linhas, letras, números ou outras formas (MONTIEL; CAPOVILlA, 2008). Os testes de Desenho são muito utilizados na avaliaçáo de NU, pois esses pacientes têm dificuldades em produzir desenhos simétricos de objetos, como borboletas e margaridas. O Teste de Desatenção Comportamental (BIT) foi idealizado para incluir os testes convencionais de negligência e os testes de habilidades diárias, a fim de fornecer informações mais abrangentes para o programa de reabilitação. É composto por seis subtestes convencionais, como bissecção de linha, tarefas de cancelamento e cópias de figuras, e nove subtestes comportamentais que refletem problemas diários, como varredura de figuras, discar telefone, ler cardápio, contar e marcar o tempo e acompanhar mapas (HARTMAN-MAEIR; KATZ, 1995).

Outra possibilidade de avaliação consiste em observar a ocorrência da NU durante a realizaçáo de tarefas rotineiras, incluindo, por exemplo, atividades relacionadas ao próprio corpo (autocuidado), à jardinagem, às compras do cotidiano (GRIEVE, 2006). Estudos conduzidos por terapeutas ocupacionais têm investigado as implicaçôes das práticas realizadas, examinando a repercussão delas na autonomia e independência dos sujeitos acompanhados (TSUKIMOTO; VALESTER, 2005).

Hagedorn (2003) fala do tratamento terapêutico ocupacional para o déficit de percepção, utilizado a Estrutura Aplicada de Referência CognitivoPerceptiva (EAR), que consiste em treinar os processos mentais "ocultos", como se situar espacialmente, reconhecer objetos e pessoas, lembrar, usar a lógica. Aborda o diagnóstico, por meio de avaliações padronizadas, o tratamento terapêutico com o uso do treino da habilidade perceptiva, e a compensação, que envolve dicas adicionais estruturando o ambiente para melhorar a percepção, como usar cores para identificar objetos e fragmentar tarefas.

$\mathrm{Na}$ Terapia Ocupacional também utilizam-se avaliações da percepção e da cognição dos referenciais da Neurociência citadas anteriormente: testes bidimensionais, como seccionar uma linha ao meio, tarefa de cancelamento de letras e números e cópia de desenho. E testes de comportamento (GRIEVE, 2006; EDMANS, 2004; TROMBLY, 2005). Em Neuropsicologia para terapeutas ocupacionais há uma discussão sobre a importância de se relacionar os 
testes convencionais com as atividades funcionais. $\mathrm{E}$ Carr e Shepherd (2008) ainda falam que esses testes comportamentais são estratégias fundamentais na reabilitação, citando outros testes relevantes como o Teste de verticalidade e o Teste de percepção auditiva.

O tratamento das deficiências de percepção pela Terapia Ocupacional descrito por Edmans (2004) baseia-se na transferência do treinamento; na integração sensorial, no desenvolvimento neurológico, no método de Affolter, no Desempenho Ocupacional, no Método Funcional e Método Dinâmico de Interação. E as estratégias utilizadas nas sessôes incluem: objetos que são colocados na linha média e, gradativamente, deslocados para o lado afetado do paciente; utilização de margens para delimitar o ambiente e os objetos; adaptaçóes do ambiente e objetos.

López, Molina e Arnaiz (2001) e Edmans (2004) orientam que no atendimento de Terapia Ocupacional as atividades devem ser decompostas para ficarem simples. Recomendam que, perante demandas funcionais específicas de atividades que englobem, por exemplo, uso de calendário, leitura, diário para memória, sempre sejam oferecidas dicas verbais, táteis e visuais, além de pistas baseadas na primeira letra, com oportunidade de repetiçáo do processo mental. Quanto à sistematização de terapias, alertam que é preciso examinar a capacidade perceptiva para AVDs e criar rotinas para a execução de cada atividade.

Considerando a importância de se investigar os resultados das terapias dirigidas para o paciente com NU, neste estudo deu-se ênfase à descrição da intervenção nos domínios da área de Terapia Ocupacional, com o objetivo de identificar o progresso do paciente com base no contínuo do seu quadro evolutivo durante o período de tratamento.

\section{Método}

A pesquisa é de caráter descritivo-analítico e tem como delineamento metodológico o estudo de sujeito único. Os dados foram coletados a partir de acompanhamento de atendimento realizado pelo serviço de Terapia Ocupacional durante um período de 10 meses. Os procedimentos de coleta de dados abrangeram o levantamento do registro de prontuário do paciente e o desenvolvimento de entrevistas semiestruturadas realizadas com a terapeuta ocupacional, com o paciente e com a cuidadora.

A pesquisa foi submetida e aprova pelo Comitê de Ética na Pesquisa da Faculdade de Filosofia e Ciências da Unesp - Campus de Marília, SP, atendendo a resolução 196/98.

\section{Resultados}

Os resultados abrangem a descrição do atendimento, contemplando os seguintes aspectos:

Dados pessoais: Paciente denominado L, com 51 anos de idade; solteiro; aposentado (profissão pintor); residente com a irmá, o cunhado e a sobrinha $S$ (identificada como sua cuidadora); escolaridade até a quarta série do ensino fundamental.

Dados clínicos: Cadeirante, diagnóstico médico de AVEh no HD, em decorrência de um aneurisma; hemiplegia e negligência unilateral esquerda; disfagia orofaríngea, disartria espástica e comportamento depressivo.

Queixa: Segundo relatos da cuidadora, o paciente necessitava de ajuda parcial para a realizaçáo de cuidados com a aparência; permanecia isolado e não manifestava interesse no envolvimento em atividades sociais.

Dados de intervençáo de Terapia Ocupacional: Foram aplicados o Teste de Cancelamento de Letras, Teste de Cancelamento de Números, Teste do Relógio e o Teste do Desenho. Na aplicação do Teste de Cancelamento de Letra foi solicitado ao paciente que circulasse a primeira letra do seu nome. L circulou todas as letras presentes no quadro (Figura 1). Entretanto, segundo os relatos da terapeuta, o paciente necessitou de pistas verbais e visuais para circular as letras dispostas no lado esquerdo do quadro.

Em relaçáo ao Teste de Cancelamento de Número foi solicitado a $\mathrm{L}$ que circulasse todos os números 1 . O paciente circulou todos os números (Figura 2). Porém apresentou as mesmas dificuldades apresentadas no Teste de Cancelamento de Letra.

$\mathrm{Na}$ aplicação do Teste do Relógio foi solicitado ao paciente que completasse o desenho do relógio. A terapeuta desenhou o círculo e os quadrantes do relógio. L não conseguiu desenhar de forma completa e correta os números em cada um dos quadrantes (Figura 3). Tal fato contribuiu para reforçar a presença da NU, relacionada à lesão do HD e afetando as funções relacionadas ao lado esquerdo do corpo. $\mathrm{O}$ paciente tentou desenhar novamente o relógio, mas também náo obteve sucesso.

Para a realização do Teste do Desenho, foi apresentado ao paciente o desenho (modelo) de uma casa (Figura 4) e foi solicitado a ele que copiasse o referido desenho da casa. L cumpriu a tarefa com dificuldade. Inicialmente foram necessárias muitas pistas da terapeuta para dirigir a atençấo do paciente 


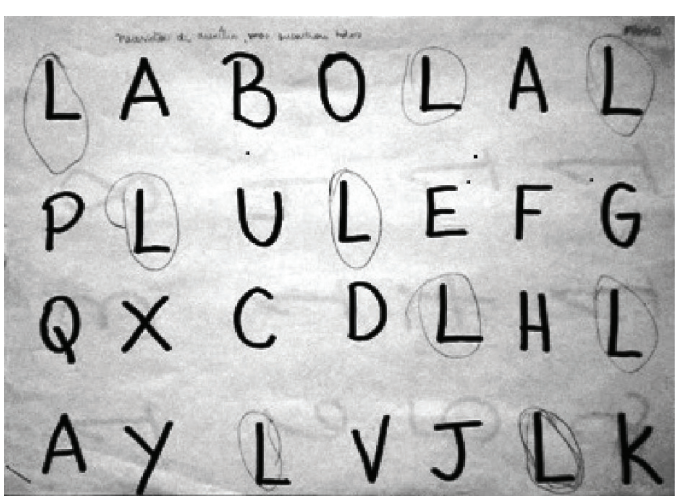

Figura 1. Teste de Cancelamento de Letra.

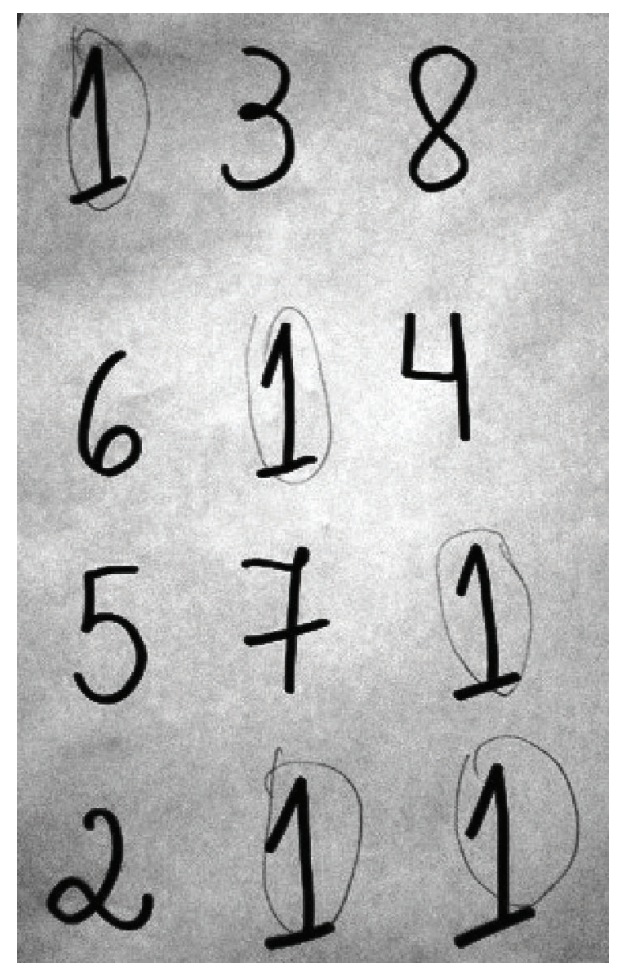

Figura 2. Teste de Cancelamento de Número.

para o lado esquerdo. A partir do teste do desenho observou-se no paciente um déficit em termos de noção espacial. O paciente ignorou o lado esquerdo do papel, desenhando apenas na parte direita, bem como negligenciou a parte esquerda do desenho. Interessante observar que $\mathrm{L}$ desenhou a parede e a porta no lado direito e o sol sob a casa (Figura 5). O que confirma o que Lundy-Ekman (2008) fala da dificuldade com desenho e com montagem, devida à diminuição da compreensão das relaçôes espaciais, chamada de apraxia de construção.

Em todos os procedimentos, a terapeuta ocupacional demonstrava a forma de posicionamento corporal adequada. Nesse sentido, Carlo e Luzo

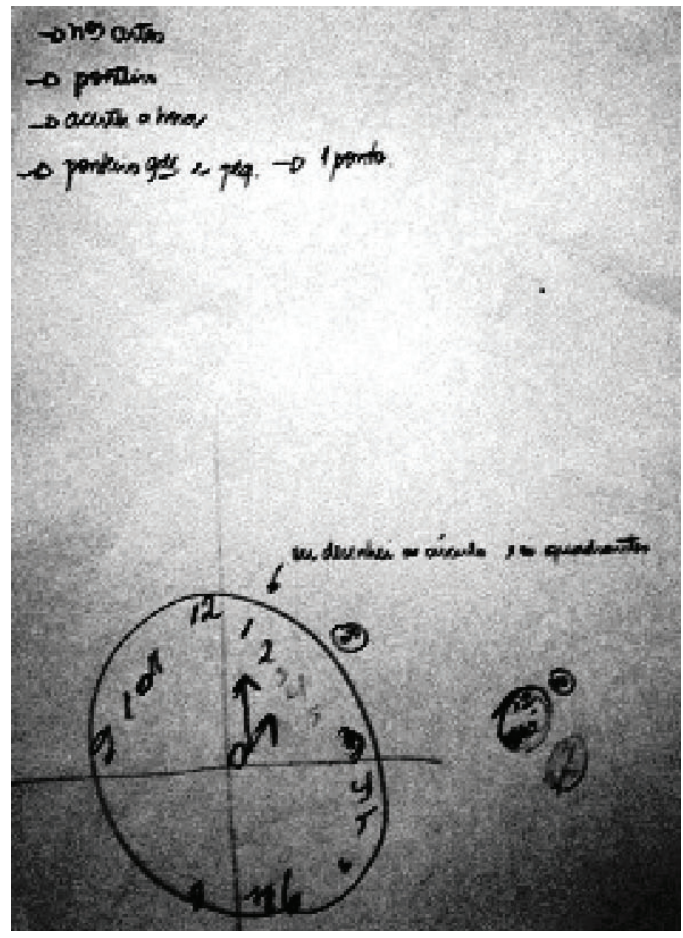

Figura 3. Teste do Relógio.

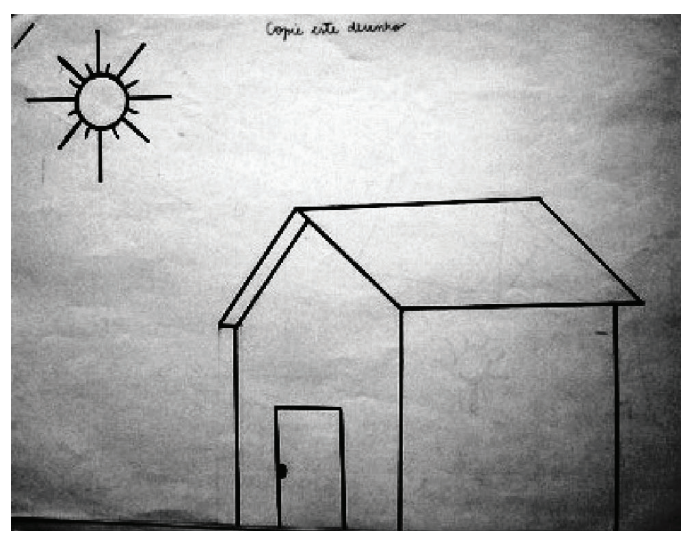

Figura 4. Teste do Desenho: modelo.

(2004) observam que a orientação sobre o posicionamento correto dos membros superiores favorece a recuperação das atividades funcionais no decorrer dos procedimentos voltados à reabilitaçáo. No caso de L observamos a necessidade de estimulação e direcionamento constantes por parte da terapeuta durante a realizaçáo das tarefas. Isso ocorreu em função do déficit de atenção apresentado pelo paciente. Tais procedimentos são consonantes com as prescriçôes de Terapia Ocupacional para controle de feedback.

Nas sessôes iniciais de terapia, L foi incentivado a relatar atividades cotidianas e ocupaçôes por ele realizadas antes do AVE. A terapeuta utilizou 
como apoio da intervenção a própria relação terapeuta-paciente e atividades expressivas (desenho livre, por exemplo). Durante a execuçáo de atividades expressivas, a terapeuta utilizou modelos para a reprodução de algumas formas ou figuras representativas da ocupação profissional do paciente (telhados, casa, portas, janelas). Durante as terapias foram oferecidas atividades que faziam parte do contexto e conhecimento do paciente, de modo que as atividades realizadas partiram do interesse de L, que optou por realizar a pintura de cenários que representavam o seu cotidiano profissional. Utilizou preferencialmente a mão direita (lado dominante), mas também foi incentivado a usar a mão esquerda em alguns momentos, com o objetivo de estimulação da mobilidade e função sensoriomotora. Foram aplicadas estratégias que estimularam o paciente a utilizar mais o lado esquerdo como, por exemplo, organizar a distribuição de materiais e ferramentas de uso na atividade do lado negligenciado, com a terapeuta posicionando-se também do lado esquerdo para a execução de comandos verbais e visuais (Figura 6). Foi utilizada órtese longa de posicionamento de punho e dedos para o membro superior esquerdo (Figura 6) e aplicaram-se procedimentos com o objetivo de aumentar gradativamente a extensão de cotovelo e melhorar a resposta sensitiva.

L foi submetido a estímulos sensoriais e perceptivos especialmente voltados à sua imagem corporal. Tais estímulos incluíam jogos e testes. Em relação à sensibilidade foram montadas atividades utilizando sagu e grãos (Figura 7). Também foram desenvolvidas atividades relacionadas à orientação temporal como, por exemplo, a confecção de um calendário (Figura 8). Em relação às funções cognitivas, como atenção e memória, $\mathrm{L}$ ainda foi estimulado mediante atividades que envolviam jogos e leitura.

A reavaliação da terapeuta ocupacional foi feita três meses após a primeira aplicação dos testes. L obteve as seguintes evoluçôes: Teste do Relógio: não desenhou os números nos quadrantes corretos; Prova dos Animais, onde a terapeuta pede para nomear 13 animais em 1 minuto: $\mathrm{L}$ nomeou cinco; aplicação do Mini Mental: score 24 (não há relato de score anterior); Construção da casa de palito de sorvete: desenhou os objetos numa localização mais satisfatória; Teste de Cancelamento: L necessitou de pistas, mas localizou tudo, senso que no primeiro, mesmo com pistas, náo localizou; Prova de leitura de frase: leu sem auxílio.

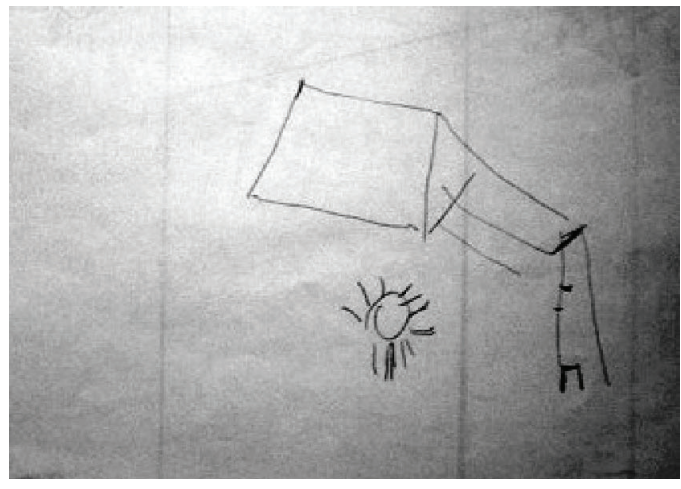

Figura 5. Teste do Desenho: cópia.

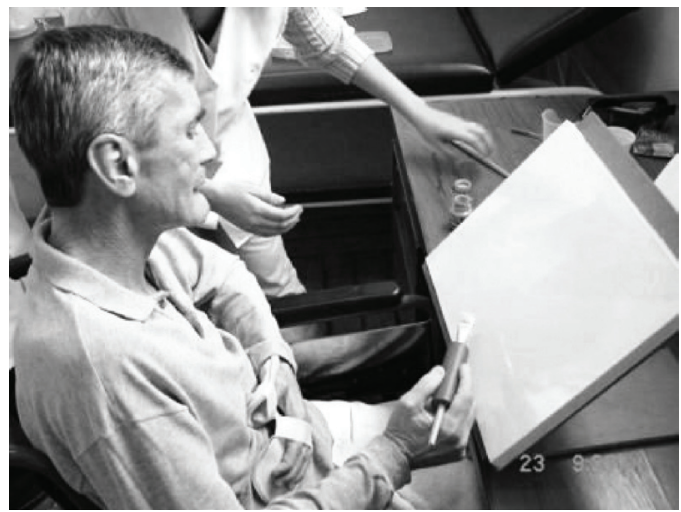

Figura 6. Estimulação visual e verbal voltados para o lado esquerdo; mão esquerda posicionada com uso de órtese.

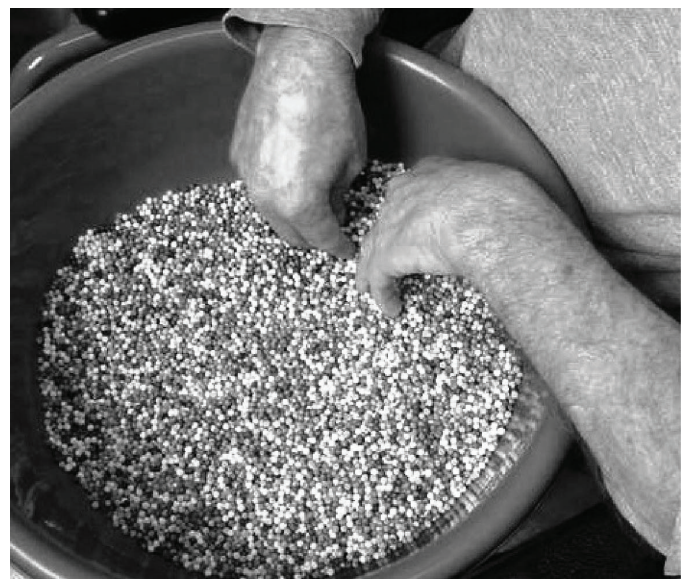

Figura 7. Atividades voltadas à estimulação sensitiva.

\section{Resultados}

Os resultados apontam para uma melhora do estado geral do paciente no decorrer das sessóes de Terapia Ocupacional.

A cuidadora (S) relatou melhora gradativa do paciente nos seis primeiros meses após o início do tratamento. Segundo ela: 


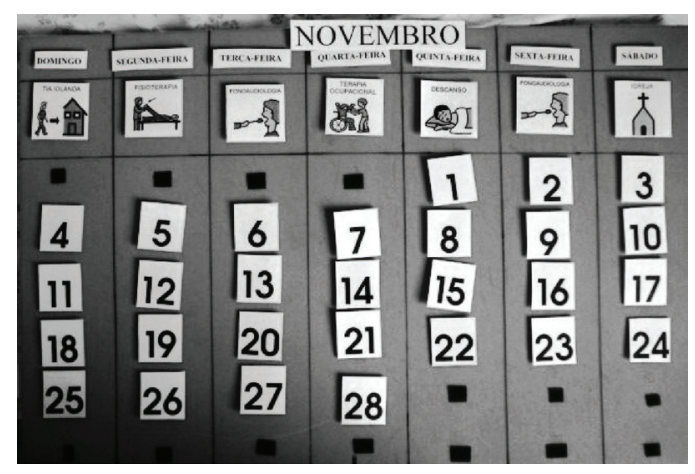

Figura 8. Atividades voltadas à orientação temporal.

É o atendimento que L mais gosta e o que mais fez ele melhorar. Em casa ele pergunta se é o dia da Terapia Ocupacional. Quando venho aqui para o atendimento da físio e se ele vê a terapeuta ocupacional, ele a chama para perguntar se ele vai ser atendido. [...] antes não olhava para o lado esquerdo, agora olha. Ele está mais feliz, ele fica alegre quando vem para o atendimento de Terapia Ocupacional.

Também, no intervalo de seis meses, o paciente percebeu sua recuperação, relatando que "sentia prazer ao fazer atividade" e que "se sentia mais alegre com a vida". Quando a pesquisadora perguntou o que ele esperava dos atendimentos de Terapia Ocupacional, L respondeu: "quero continuar fazendo TO porque gosto".

Essa motivação apresentada pelo paciente aponta para a importância da Terapia Ocupacional no processo de reabilitação. Esse feedback foi importante para a avaliaçáo dos procedimentos e dos resultados do tratamento realizado. Entretanto, nos dois meses seguintes se observou uma estagnação da evolução de L. Nesse período crítico, L não seguia as orientaçóes da terapeuta ocupacional. Nas entrevistas realizadas com a cuidadora e com o próprio paciente, foram evidenciados desânimo, falta ou dificuldade de concentraçáo, sono e resistência para realizar atividades. Pôde-se observar ainda que o estado físico, sobretudo a incapacidade de andar, deixava o paciente irritado e ansioso. Também foram relatadas oscilaçóes em relação à pressão arterial.

\section{Discussão}

As observaçóes e dados levantados durante a realização do estudo corroboram, de forma geral, os dados e as interpretaçôes decorrentes de pesquisas acerca da NU (NAPOLITANO; TRIACA, 2007; MESULAM, 1999; CAMPOS;
SANTOS; XAVIER, 1997). Após AVE no HD, L apresentou dificuldades para registrar, integrar ou responder a eventos provenientes do hemicorpo ou hemiespaço contralateral à lesão cerebral. (TSUKIMOTO; VALESTER, 2005; HEILMAN; WATSON; VALENSTEIN, 1993; HEILMAN; VALENSTEIN; WATSON, 1994). O paciente também apresentou déficits perceptivos, bem como recaída de comportamento depressivo; eventos esses também coerentes com os quadros de SNU (CARR; SHEPHERD, 2008; DOBKIN, 2005; GRIEVE, 2006; EDMANS, 2004).

Quanto à recaída observada durante o tratamento, é importante salientar que tal evento não é raro em pacientes com SNU. Lovo (2006), por exemplo, explica que a NU é um distúrbio que predispóe a um prognóstico desfavorável no processo de reabilitação, mas os fatores sáo variados, de acordo com a idade, o dano cognitivo, o dano perceptual, a depressão, a incontinência e as habilidades funcionais pré-AVE.

A dificuldade cognitiva apresentada por L relacionava-se basicamente aos mecanismos de atençáo, comprometimento esse coerente com o quadro de SNU (SWAN, 2001; MESULAM, 1994, 1985).

Existem basicamente duas hipóteses acerca do funcionamento do sistema nervoso (SN) para explicar fenômenos, como, por exemplo, o da NU (CAMPOS; SANTOS; XAVIER, 1997; XAVIER, 1993). A primeira defende a ideia de que o $S N$ funcionaria a partir de um processamento serial, ou seja, seguiria, em termos de processamento de informação, uma sequência de estágios, que culminariam em um centro privilegiado responsável pelo aparecimento da consciência. A segunda apoia-se na concepção de que o processamento estaria distribuído por todo o sistema nervoso. De acordo com essa segunda hipótese e conforme os autores, diferentes módulos de processamento trabalhariam em paralelo e o resultado dessa configuração de atividades nos diversos módulos determinaria a sensação de consciência. Assim, os fenômenos relacionados à SNU não deveriam ser atribuídos à perda de determinado repertório cognitivo, mas sim ao excessivo controle do comportamento, determinado pelo córtex parietal esquerdo, o qual permaneceu intacto.

Os dados levantados nas entrevistas realizadas com a cuidadora e o paciente indicaram que o tratamento de Terapia Ocupacional propiciou uma melhora na qualidade de vida geral do paciente. A partir dos dados do prontuário do paciente e a partir da entrevista com a terapeuta, houve indicativo de que a intervenção realizada contribuiu para: aumentar a autoestima do paciente; diminuir a negligência tanto 


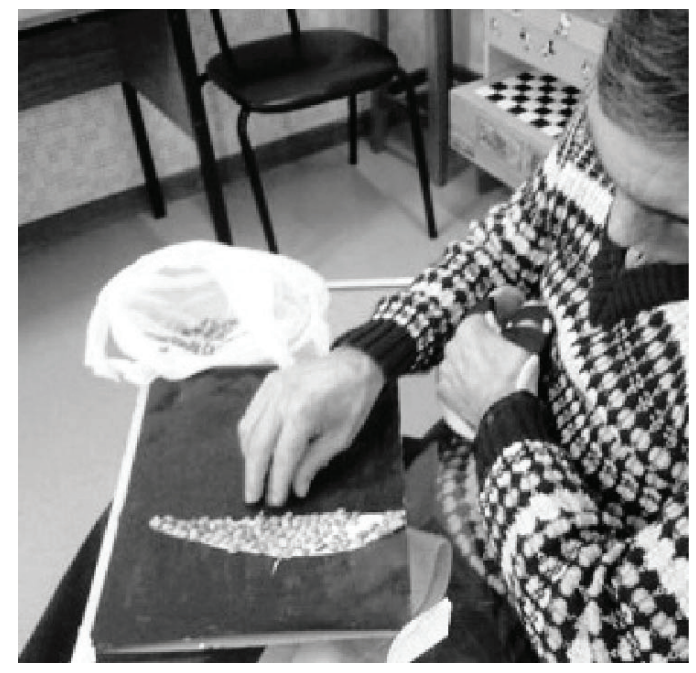

Figura 9. Paciente após 10 meses de tratamento.

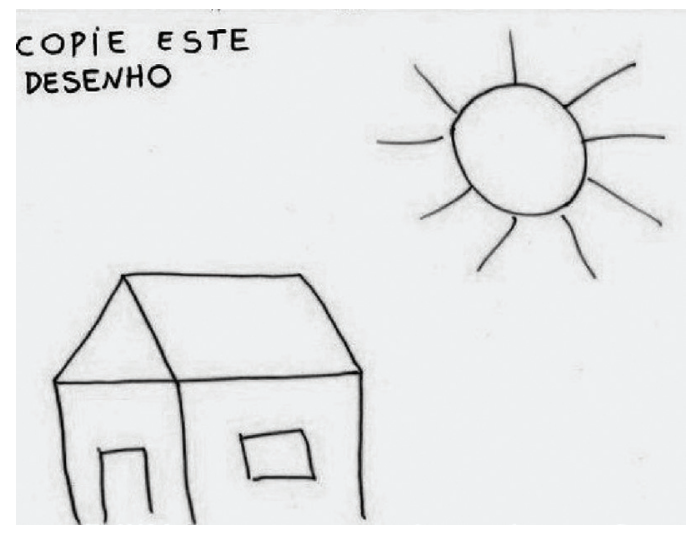

Figura 10. Teste do Desenho.

pessoal quanto espacial; melhorar o posicionamento dos membros superiores com o uso de órtese, melhorar a sensibilidade tátil e proprioceptiva. A estratégia de intervenção, que enfatizou os contextos rotineiros e habituais do paciente, gerou motivaçáo e assegurou o real envolvimento e a participação dele no tratamento, o que ficou evidenciado pelo engajamento do paciente na realização das atividades, principalmente na sua evoluçáo em termos de recuperação funcional.

O impacto das intervençóes de Terapia Ocupacional sobre a motivaçáo dos pacientes tem sido observado nos estudos conduzidos por esses profissionais. O estudo de Carin-Levy et al. (2009), que investigou os resultados das intervençóes de conduta educacional em relação à prática de exercícios e relaxamento para os pacientes com sequelas de AVE, também identificou, por meio de entrevistas semiestruturadas realizadas de seis a 18 meses após o término da programação, cinco categorias temáticas de impacto favorável: prazer de participar das aulas, motivação, qualidade de vida autopercebida, empowerment e adesão das práticas a longo prazo.

Após 10 meses de tratamento, o paciente recuperou seu interesse pelas atividades, evidenciando-se melhoras expressivas no quadro de negligência unilateral, conforme ilustram as imagens (Figuras $9 \mathrm{e}$ 10), feitas após 10 meses de tratamento. Observou-se, após esse período, uma diminuição na agnosia de contorno e uma redução do distúrbio em relação à apraxia de construção, o que resultou em melhora na capacidade executiva, na identificação de estímulos do lado esquerdo do corpo e na percepção em relação ao espaço.

Apesar da evoluçáo satisfatória apresentada pelo paciente, conforme observado nesse estudo, acreditamos que seria importante o monitoramento da alta do paciente, tendo em vista os episódios de recaída, em funçấo da vulnerabilidade psicológica dele. Nesse sentido é importante ressaltar que o processo de reabilitação de indivíduos com SNU nâo deve restringir-se apenas ao atendimento isolado de um tipo de terapia voltada ao processo de reabilitação (CARLO; LUZO, 2004; SOARES, 2003). Além disso, acrescentamos que no processo de reabilitação se faz necessário a elaboração de um programa que não ignore o contexto de vida do paciente, bem como observe o envolvimento do(s) cuidador(es) familiar(es).

\section{Referências}

AMERICAN OCCUPATIONAL THERAPY ASSOCIATION - AOTA. Estrutura da prática da terapia ocupacional: domínio e processo. 2. ed. Revista Triângulo, Uberaba, v. 3, n. 2, p. 57-147, 2010.

BAILEY, M. J.; RIDDOCH, M. J. Hemineglect. Part 1: the nature of hemineglect and its clinical assessment in stroke patients: an overview. Physical Therapy Review, London, v. 4, n. 2, p. 67-75, 1999.

BEAR, M. F.; CONNORS, B. W.; PARADISO, M. A. Neuroscience: exploring the brain. 3rd ed. Philadelphia: Lippincott Willians and Wilkins, 2006.

BONITA, R.; SOLOMON, N.; BROAD, J. B. Prevalence of stroke and stroke-related disability: estimates from the Auckland Stroke Studies. Stroke, Dallas, v. 28, n. 10, p. 1898-902, 1997.

CABRAL, N. L. et al. Epidemiologia dos acidentes cerebrovasculares em Joinville, Brasil: estudo institucional. Arquivos de Neuro-Psiquiatria, São Paulo, v. 55, n. 3A, p. 357-63, 1997.

CAMPOS, A.; SANTOS, A. M. G.; XAVIER, G. F. A consciência como fruto da evolução e do funcionamento do sistema nervoso. Psicologia USP, São Paulo, v. 8, n. 2, p. 181-226, 1997. 
CARDOSO, L. et al. Heminegligência e reabilitação cognitiva: um relato de caso. Jornal Brasileiro de Psiquiatria, Rio de Janeiro, v. 54, n. 4, p. 340-344, 2005.

CARR, J.; SHEPHERD, R. Reabilitação neurológica: otimzando o desempenho motor. São Paulo: Manole, 2008.

CARIN-LEVY, G. et al. The psychosocial effects of exercise and relaxation classes for persons surviving a stroke. Canadian Journal of Occupation Therapy, Toronto, v. 76, n. 2, p. 73-80, 2009.

CARLO, M. M. R. P.; LUZO, M. C. M. Terapia ocupacional: reabilitação física e contextos hospitalares. São Paulo: Roca, 2004.

CUBELLI, R. et al. Different patterns of dissociation in unilateral spatial neglect. Brain and Cognition, New York, v. 15, n. 2, p. 139-59, 1991.

DOBKIN, B. H. Clinical practice.rehabilitation after stroke. The New England Journal of Medicine, Boston, v. 11, n. 352, p. 1677-1684, 2005.

DOYLE, P. J. Measuring health outcomes in stroke survivors. Archives of Physical Medicine and Rehabilitation, Philadelphia, v. 83, n. 12, p. 539-43, 2002. Supplement 2 .

EDMANS, J. Terapia Ocupacional e derrame cerebral. São Paulo: Santos, 2004.

FONSECA, R. P. et al. Alterações cognitivas, comunicativas e emocionais após lesão hemisférica direita: em busca de uma caracterização da Síndrome do Hemisfério Direito. Psicologia USP, São Paulo, v. 17, n. 4, p. 241-262, 2006.

GAZZANIGA, M. S.; HEATHERTON, T. F. Ciência psicológica: mente, cérebro e comportamento. Porto Alegre: Artmed, 2005.

GAZZANIGA, M. S.; IVRY, R. B.; MANGUN, G. R. Neurociência cognitiva: a biologia da mente. 2. ed. Porto Alegre: Artmed, 2006.

GILES, M. F.; ROTHWELL, P. M. Measuring the prevalence of stroke. Neuroepidemiology, New York, v. 30, n. 4, p. 205-206, 2008.

GRIEVE, J. Neuropsicologia em Terapia Ocupacional: exame da percepção e cognição. 2. ed. São Paulo: Santos, 2006. GUIMARÃES, R. B.; GUIMARÃES, R. B. Validação e adaptação cultural para língua portuguesa de escalas de avaliação funcional em doenças cerebrovasculares: uma tentativa de padronização e melhoria da qualidade de vida. Revista Brasileira de Neurologia, Rio de Janeiro, v. 40, n. 3, p. 5-13, 2004.

HAGEDORN, R. Fundamentos para a prática em terapia ocupacional. 3. ed. São Paulo: Roca, 2003.

HARTMAN-MAEIR, A.; KATZ, N. Validity of the Behavioral Inattention Test (BIT): relationships with functional tasks. American Journal of Occupational Therapy, New York, v. 49, n. 6, p. 507-516, 1995.

HEILMAN, K. M.; VALENSTEIN, E.; WATSON, R. T. The what end how of neglect. Neuropsychological Rehabilitation, Hove, v. 4, n. 2, p. 133-139, 1994.

HEILMAN, K. M.; WATSON, R. T.; VALENSTEIN, E. Neglect and related disorders. In: HEILMAN, K.
M.; VALENSTEIN, E. (Eds.). Clinical neuropsychology. New York: Oxford University Press, 1993. p. 279-336.

HELENE, A. F.; XAVIER, G. F. A construção da atenção a partir da memória. Revista Brasileira de Psiquiatria, São Paulo, v. 25, p. 12-20, 2003. Suplemento 2.

KANDEL, E. R.; SCHWARTZ, J. H.; JESSEL, T. M. Fundamentos da neurociência e do comportamento. Rio de Janeiro: Guanabara Koogan, 2000.

LAVINSKY, A. E.; VIEIRA, T. T. Processo de cuidar de idosos com acidente vascular encefálico: sentimentos dos familiares envolvidos. Acta Scientiarm Health Sciences, Maringá, v. 26, n. 1, p. 41-45, 2004.

LESSA, I. Epidemiologia das doenças cerebrovasculares no Brasil. Revista da Sociedade de Cardiologia do Estado de São Paulo, São Paulo, v. 9, n. 4, p. 509-518, 1999.

LÓPEZ, B. P.; MOLINA, P. D.; ARNAIZ, B. N. Conceptos fundamentales de terapia ocupacional. Madrid: Médica Panamericana, 2001.

LOVO, T. M. A. Anosognosia: imagem corporal na hemiplegia. 2006. 135 f. Dissertação (Mestrado em Educação Física)-Faculdade de Educação Física, Universidade Estadual de Campinas, Campinas, 2006. p. 73-83.

LUNDY-EKMAN, L. Neurociência: fundamentos para a reabilitação. 2. ed. Rio de Janeiro: Elsevier, 2004.

LUNDY-EKMAN, L. Neurociência: fundamentos para a reabilitação. 3. ed. Rio de Janeiro: Elsevier, 2008.

MARK, V. W. Acute versus chronic functional aspects of unilateral spatial neglect. Frontiers in Bioscience, Florida, v. 1, n. 8, p. 172-189, 2003.

MENDONÇA, F. F.; GARANHANI, M. L.; MARTINS, V. L. Cuidador familiar de sequelados de acidente vascular cerebral: significado e implicaçóes. Physis: Revista de Saúde Coletiva, Rio de Janeiro, v. 18, n. 1, p. 143-158, 2008.

MESULAM, M. M. Principles of behavioral neurology. Philadelphia: F.A. Davis Company, 1985.

MESULAM, M. M. Spatial attention and neglect: parietal, frontal and cingulate contributions to the mental representation and attentional targeting of salient extrapersonal events. Philosophical Transactions of the Royal Society of London: Series B, London, v. 354, n. 1387, p. $1325-1346,1999$.

MESULAM, M. M. The multiplicity of neglect phenomena. Neuropsychological Rehabilitation, Hove, v. 4, n. 2, p. 173-176, 1994.

MONTIEL, J. M.; CAPOVILlA, A. G. S. Teste de atenção por cancelamento: análise de critérios de correção. Integração, São Paulo, v. 14, n. 54, p. 288-296, 2008.

MOURA, E.; SILVA, P. Fisioterapia: aspectos clínicos e práticos da reabilitação. Rio de Janeiro: Artes Médicas, 2005.

NAPOLITANO, R. A.; TRIACA, T. P. Hemineglicência: um estudo de caso. Arquivos de Ciências da Saúde da UNIPAR, Umuarama, v. 11, n. 2, p. 137- 143, 2007. 
PETRILLI, S. et al. Prognostic factors in the ability to walk after stroke. Journal of Stroke and Cerebrovascular Diseases, Philadelphia, v. 11, n. 6, p. 330-335, 2002.

PIERCE, S. R.; BUXBAUM, L. J. Treatments of unilateral neglect: a review. Archives of Physical Medicine and Rehabilitation, Chicago, v. 83, n. 2, p. 256-68, 2002.

PLUMMER, P.; MORRIS, M. E.; DUNAI, J. Assessment of unilateral neglect. Physical Therapy, Alexandria, v. 83, n. 8, p. 732-740, 2003.

ROBERTSON, I. H.; MARSHALL, J. C. (Eds.). Unilateral neglect: clinical and experimental studies. Hove: Lawrence Erlbaum, 1993.

RODGERS, H. Risk factors for first-ever stroke in older people in the North East of England: a populationbased study. Stroke, Dallas, v. 35, n. 1, p. 7-11, 2004.

SANTOMÉ, A.; MARTÍN-LOECHES, M.; FRANK, A. Mecanismos neurofisiológicos del síndrome de negligencia unilateral. Revista de Psicología General y Aplicada, Madrid, v. 51, n. 3-4, p. 443-454, 1998.

SAPOSNIK, G.; DEL BRUTTO, O. H. Stroke in South America: a systematic review of incidence, prevalence and stroke subtypes. Stroke, Dallas, v. 34, n. 9, p. 2103-218, 2003.

SARTI, C. et al. International trends of disease study. Stroke, Dallas, v. 31, p. 1588-1601, 2000.

SCHAU, B. et al. Development and validation of a model to estimate stroke incidence in a population. Journal of
Stroke and Cerebrovascular Diseases, Philadelphia, v. 12, n. 1, p. 22-28, 2003.

SILVA, C.; MARTINS, J. Negligência unilateral: implicações no processo de reabilitação. Revista de Fisioterapia da Universidade de São Paulo, São Paulo, v. 7, n. 1-2, p. 16-24, 2000.

SILVA, F. Acidente vascular cerebral isquêmico. Prevenção: aspectos actuais. É preciso agir. Medicina Interna, Bucuresti, v. 11, n. 2, p. 99-108, 2004

SOARES, E. Desenvolvimento e dificuldades da concepção localizacionista de funçóes mentais: uma análise a partir de Merleau-Ponty. 2003. 189 f. Tese (Doutorado em Psicologia, Neurociências e Comportamento)-Universidade de São Paulo, São Paulo, 2003.

STOKES, M. Neurologia para fisioterapeutas. São Paulo: Premier, 2000.

SWAN, L. Unilateral spatial neglect. Physical Therapy, Albany, v. 81, n. 9, p. 1572-1580, 2001.

TROMBLY, C. A. Terapia ocupacional para disfunçóes físicas. São Paulo: Santos, 2005.

TSUKIMOTO, D. R.; VALESTER, G. A. A influência da negligência unilateral no desempenho de atividades de vida cotidiana: relato de 3 casos. Acta Fisiatrica, São Paulo, v. 12, n. 3, p. 108-114, 2005.

XAVIER, G. F. A modularidade da memória e o sistema nervoso. Psicologia USP, São Paulo, v. 4, n. 1-2, p. 61-115, 1993.

\section{Contribuição dos Autores}

Tamara Pereira de Oliveira: Concepção do texto, organização de fontes e/ou análises, redação do texto. Rita de Cássia Tibério Araújo e Edvaldo Soares: Orientação, análise e revisão de texto.

\section{Notas}

${ }^{1}$ Esta pesquisa é resultado de estudo de caso - análise de intervençôes. A pesquisa foi submetida e aprova pelo Comitê de Ética em Pesquisa em Saúde da Faculdade de Filosofia e Ciências - FFC - Unesp - Campus de Marília, SP, conforme ofício número 1951/2008. Todos os procedimentos éticos necessários foram realizados. 